\title{
Study of Dipole Antennas for Application in Backscatter Radio Links
}

\author{
Raquel Aline A. Rodrigues, Francisco M. de Assis, Edmar C. Gurjão, Rômulo R. M. do Valle
}

\begin{abstract}
This paper presents a method to measure backscattering signal from antennas using a network analyzer connected to an anechoic chamber. We analyze the backscattered signals of two types of dipoles: wire dipole at the operating frequency of $1.8 \mathrm{GHz}$ and microstrip dipole operating frequency of $900 \mathrm{MHz}$. The experimental results showed that the receiving antenna dipole wire showed a better reflection of the backscattered signal compared to the microstrip dipole.
\end{abstract}

Keywords- backscattered signal; dipole antenna of $2 / 2$; microstrip dipole; anechoic chamber.

\section{INTRODUCTION}

The wave reflection intentionally issued on an object has been a topic of active study since the development of Radar in 1930 [1]. In addition, since Stocman proposed the concept of backscatter modulation for communication in 1948, continuous research and development are performed in the industry and academia. Successful applications of this technique are Frequency Identification (RFID), passive sensors and data storage liabilities [2].

The applications already used in commercial products of this technique include parcel tracking, temperature sensors, inventory control, position location, memory cards passive, and so on. The growth of radio backscatter technology contributes to applications requiring communication with low power consumption.

In Reference [3] is proposed a backscatter radio for wireless sensor networks. They presented a complete model of backscatter radio link, the system architecture and a set of techniques for extracting information from data for each sensor. In [4], it is presented a chipless RFID where data are reading using electromagnetic waves and where the medium encoding the data is completely passive. The work in [5] introduced a new approach to communication backscatter. The nodes of the communication system are treated as if they were a single virtual transmitter. It can then be identified as a code collision bits transmitted by the nodes.

Aiming to use the backscatter technique for identification and sensing, more specifically in Chipless RFID system, this paper presents a study with two types of dipole antennas: wire dipole of $\lambda / 2$ and microstrip dipole operating with vertical polarization at frequencies of $1.8 \mathrm{GHz}$ and $1.7 \mathrm{GHz}$.

\section{THEORY OF BACKSCATTERING}

Backscatter is a broad class of systems that communicate using reflected electromagnetic waves, the technology is propelled in many applications, such as the already mentioned

Raquel Aline Araújo Rodrigues, Department of Electrical Engineering, PhD Student at the Federal University of Campina Grande (UFCG), Campina Grande- PB, Brazil and Federal University of Piauí, Teresina- PI, Brazil, Francisco Marcos de Assis, Edmar Candeia Gurjão and Rômulo Raimundo Maranhão do Valle, Department of Electrical Engineering, UFCG, Campina Grande- PB, Brazil E-mails: raquel.rodrigues@ufpi.edu.br, fmarcos@dee.ufcg.edu.br, rvalle@dee.ufcg.edu.br, ecandeia@dee.ufcg.edu.br. This work was partially supported by Capes. radio frequency identification (RFID) and passive sensors [2]. The flow of current in a transmitting antenna leads to an induced voltage in the receiving antenna. If the antenna is connected to a load that has low impedance (resistance), a current will be induced in the receiving antenna [1].

In Figure 1, the smallest load possible short-circuit, is illustrated. This induced current is not different from the current on the antenna transmission, such current leads irradiation, because according to the reciprocity principle any structure that receives a wave can make a way back to the transmitting antenna, inducing a voltage and therefore, produce a signal that can be detected: a backscattered signal. On the other hand, if a load with large impedance is between the antenna and the earth, little or no current is induced.

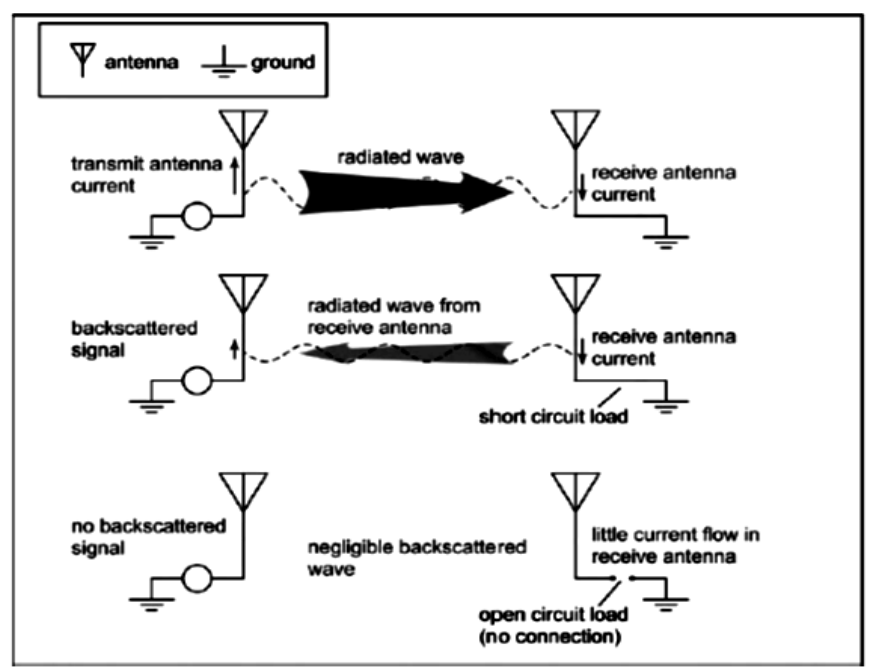

Fig. 1. Simplified physics of backscatter signaling [1]

In order to implement a backscattering scheme, there are two components, a reader that transmit a signal and receive the backscattered wave, and a tag that reflects the signal. For example, in a passive RFID system, the transmitter sends a continuous wave during the time that the receiver is waiting for the signal from the tag. RFID uses radio specialized components with known circulators that allow only reflected signals reach the receiver. However, in a system of single antenna, the signal transmitted from the reader is folded in its own antenna back to the receiver, antenna and transmitted wave is reflected from any nearby objects, adding the tag to be located (Fig. 2). 


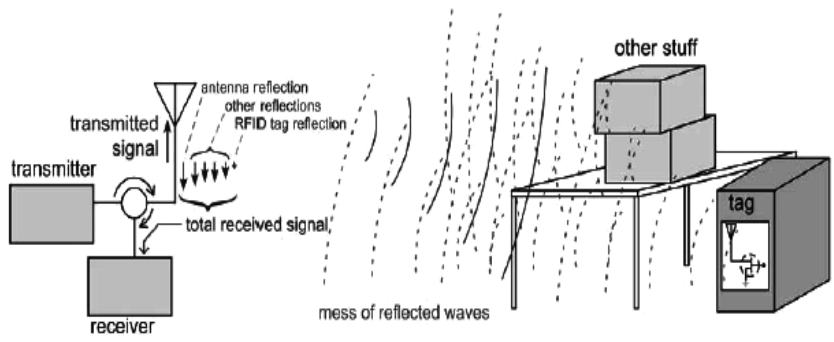

Fig. 2. Realistic environments create many reflected waves in addition to that from the wanted tag [1].

If two antennas are used (one to transmit and the other to receive) there is a signal transmitted directly from one to another, as well as spurious reflections shown in Figure 3. The total signal is the vector sum of all such contributions, most of which are much larger than the signal from the desired tag.

\section{ANTENNAS FOR BACKSCATTERING}

The design for transmitting and receiving antennas is one of the key factors for a backscatter radio link. The reading range and detection accuracy are directly dependent on the performance of these antennas.

As an example of the analysis of systems that use backscatter, consider RFID systems. For the development of a measurement system with RFID technology, it is necessary four basic components: a transponder (more commonly just called the tag), the transceiver (more commonly called a reader), an antenna attached to the reader to communicate with transponders and a reader interface layer, or middleware. The optimized antenna design will benefit the RFID systems with longer reading range, better detection accuracy, lower fabrication cost, and simple system configuration and implementation [6].

RFID technology can be classified into systems where the transponder contains a microcontroller and systems where the transponder is "chipless" [7].

Reference [8] proposed a chipless RFID transponder consisting of a printed $n$-element dipole array with different gap distances between the elements. In [9], two configurations of the developed chipless passive tags was presented. The first configuration consists in a tag with a sensor connected as a load to a microstrip triangular antenna, the second configuration consists in an antenna, microstrip transmission line (ID generation circuit), and a sensor, which is integrated, as a capacitive load to the transmission line. In [10] exposed a chipless RFID system, which was proposed UWB disc-loaded monopole antennas for tag and log periodic dipole antennas for the reader with a description of the design and results of the antenna characteristics.

As part of research on antennas for chipless RFID tags and readers, example of a backscatter radio link, in this present study were analyzed two types of dipole antennas, fabricated in LEMA-Laboratory of Applied Electromagnetics and Microwave- DEE/ CEEI /UFCG.

The dipoles choice is due to its simplicity of construction, and together with the monopoles are the most used types of antennas used in mobile wireless communication systems. The characteristics of gain and radiation efficiency of these elements are strongly influenced by its electric length, which is related to the operating frequency [11].

\section{A. Dipole Antenna of $\lambda / 2$}

Two wire dipole antennas were modeled with length $\lambda / 2$ to the frequency of $1.8 \mathrm{GHz}$ (Fig. 3). In the next step, we simulated the operation of the antenna with the software CST Microwave Studio, and checked in the laboratory, the results are shown in Figure 4.

Each dipole antenna was listed for identification, fed with power of $1 \mathrm{~mW}$ and individually calibrated and tested scattering parameters and the Smith Chart, so that these could be considered approximately equal. The dipoles present input impedances $Z_{1}=46+\mathrm{j} 6.5$ and $Z_{2}=50+\mathrm{j} 14.7$.

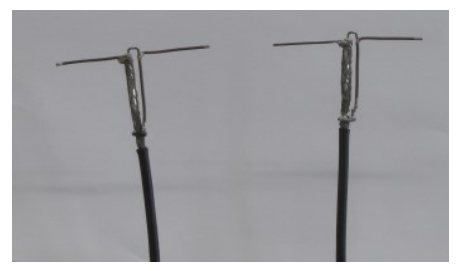

Fig. 3. Photos of the dipole antennas built-in LEMA.

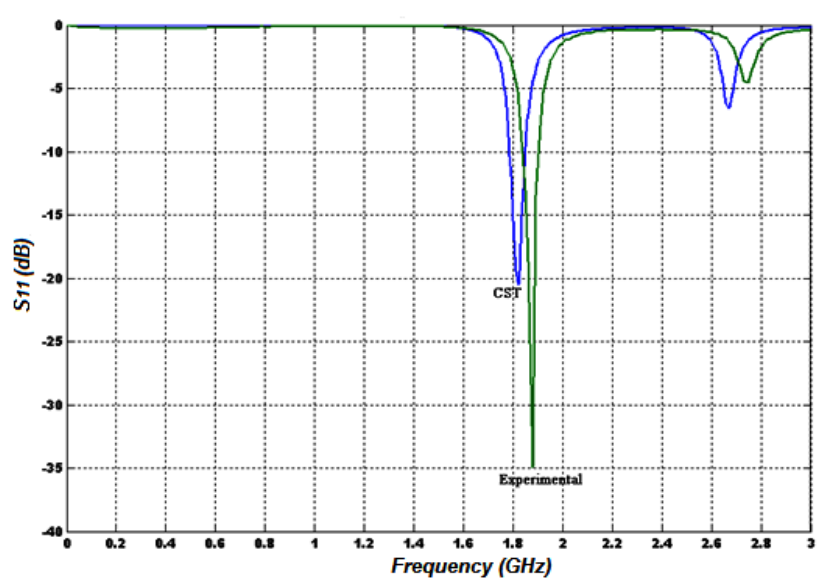

Fig. 4. Scattering parameter S11 of the dipole antenna of $\lambda / 2$.

\section{B. Microstrip Dipole Antenna}

A planar dipole antenna was modeled in CST Microwave Studio with dimensions $10 \times 10 \mathrm{~cm}$ and the resonance frequency of $900 \mathrm{MHz}$, as can be seen in Figure 5.

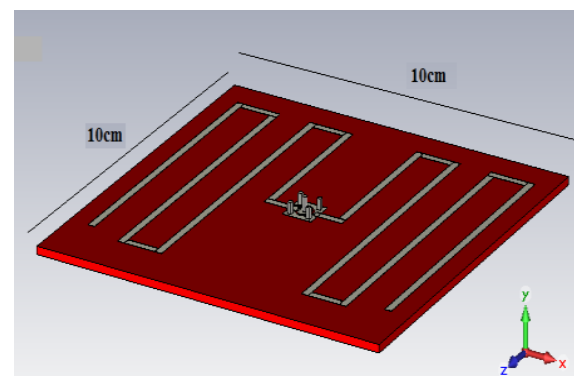

(a) Dimensions of the simulated microstrip dipole. 


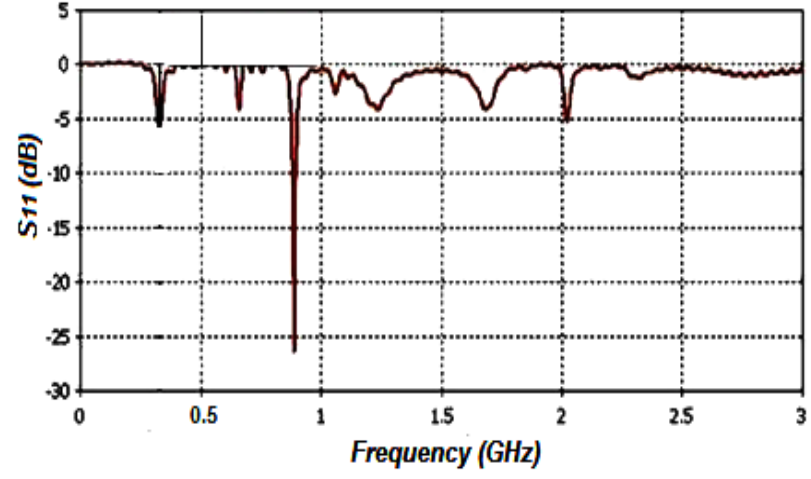

(b) Reflection coefficient of the microstrip dipole simulated.

Fig. 5. Microstrip dipole modeled in software CST MICROWAVE STUDIO.

The following modeling step was the construction of two planar dipoles in the laboratory with according to Figure 6 . The dipoles were properly identified as antenna 1 and antenna 2. Using a Vector Network Analyzer Model Rohde \& Schwarz ZBV-20 and a transmitting log-periodic Rohde \& Schwarz antenna model 12-144980 with antenna factor $28.8 \mathrm{~dB}$ were observed scattering parameters (S11 and S21). Smith Chart and irradiation diagrams of the dipoles, so that they might be considered approximately equal.
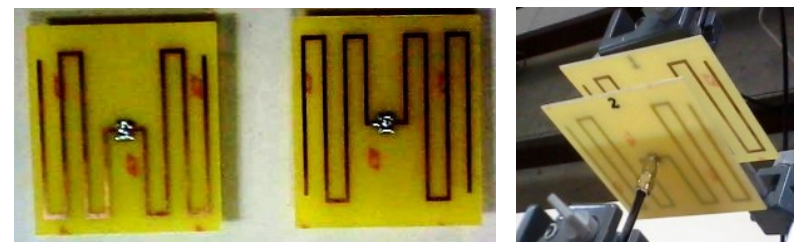

Fig. 6 Photos of microstrip dipoles built at LEMA / DEE / UFCG.

\section{The Far-field}

The measurements performed in this article with the dipoles in study were conducted in the region of the distant fields of antennas determined from the following conditions [12]:

$$
\begin{aligned}
r & =\frac{2 D^{2}}{\lambda} \\
r & =5 D \\
r & =1,6 \lambda
\end{aligned}
$$

Where: $r=$ distance from the antenna to the far field $[\mathrm{m}]$; $\mathrm{D}=$ dimension of the antenna elements $[\mathrm{m}], \lambda=$ wavelength in the operating frequency $[\mathrm{m}]$.

For wire dipole of $\lambda / 2$ : substituting $\lambda=0.17 \mathrm{~m}$ and $\mathrm{D}=\lambda / 2=0.085 \mathrm{~m}$ (at $1.8 \mathrm{GHz}$ ) comes to air $5 D=42.5 \mathrm{~cm}$.

For microstrip dipole: at $900 \mathrm{MHz}$ and $\mathrm{D}=10 \mathrm{~cm}$, comes to air $1,6 \lambda=53.3 \mathrm{~cm}$.

\section{MEASUREMENT Methodology}

There exist various techniques for measuring signal backscattering from antennas [1], [13], [14]. In this paper we used a method based on a single port network analyzer measurement using the experimental setup shown in Figure 7 [15].

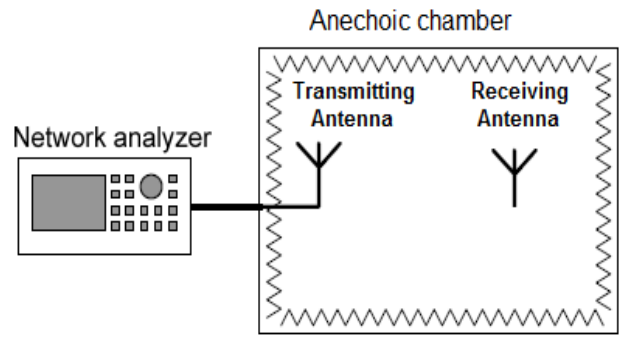

Fig.7 Experimental setup for measuring signal backscattering

The return loss of the transmitting antenna is initially measured with the network analyzer with the anechoic chamber empty, without the receiving antenna. The measured value includes the effects of input port mismatch and internal reflections inside the anechoic chamber. Then, the return loss of the antenna transmission is measured again in the presence of receiving antenna in the anechoic chamber. Thus, we compare the values of return loss for the two situations, observing the reflection wave caused by the receiving antenna, which is the backscattered signal.

We performed the measurements in anechoic chamber using the transmitting antenna connected to the Vector Network Analyzer Model Rohde \& Schwarz ZBV-20 HP8719C with $1 \mathrm{~m}$ coaxial cable.

Measurements were performed in two stages: the first with two wire dipoles of $\lambda / 2$ (Fig. 8) and the second with two microstrip dipoles (Fig. 9). In both stages, each pair of antennas was used as transmitting antenna and receiving antenna.

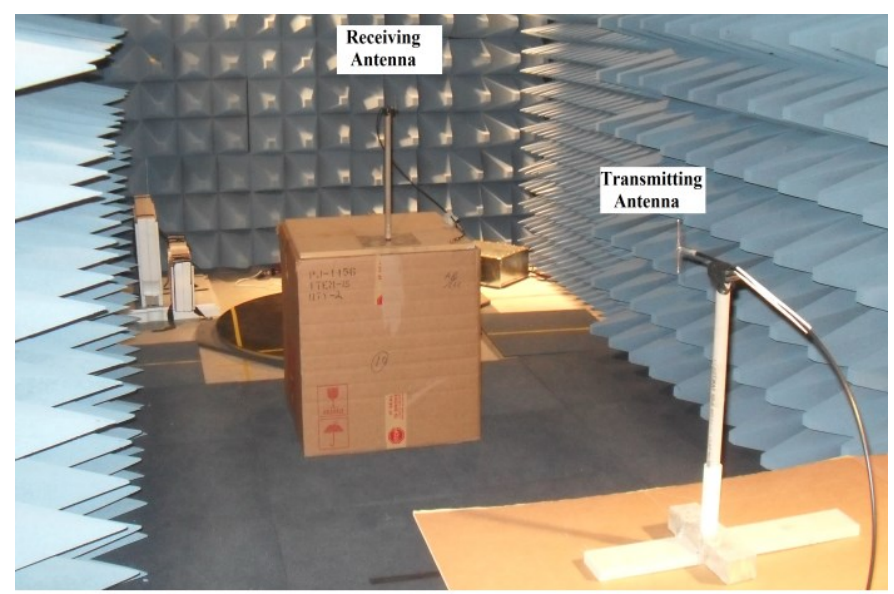

Fig. 8 The anechoic chamber with wire dipoles of $\lambda / 2: 2 \mathrm{~m}$ distance between the transmitting antenna and the receiving antenna.

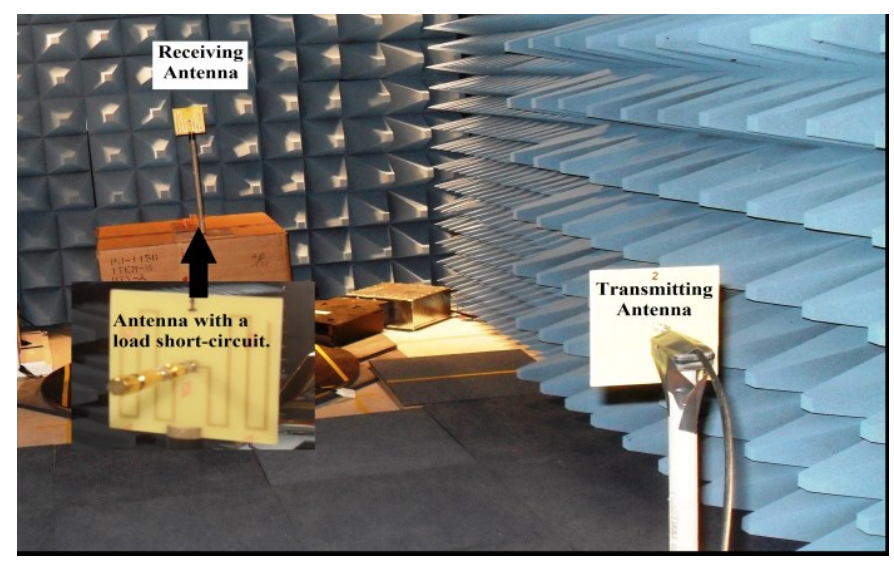

Fig. 9 The anechoic chamber with microstrip dipoles: $3 \mathrm{~m}$ distance between the transmitting antenna and the receiving antenna. 
Measurements were performed in an anechoic chamber for three distances between the transmitter and receiver antennas: $1 \mathrm{~m}, 2 \mathrm{~m}$ and $3 \mathrm{~m}$ in the far-field region. The antennas were placed on supports and cardboard box $1 \mathrm{~m}$ soil. The linearly polarized transmitting antennas were fed with $1 \mathrm{~mW}$ of power and the linearly polarized receive antennas were tested with a load short-circuit and a matched load to the three aforementioned distances.

\section{RESULTS}

In Tables 1 and 2 there are the return loss of the transmitting antennas wire dipole and microstrip dipole respectively, their resonance frequencies. The antennas were within the anechoic chamber and S11 values were measured with and without receiving antennas, the latter connected to a load short circuit or to a matched load.

It was observed that for wire dipole transmitting antenna, the value of S11 was greater in the presence of receiving antenna at a distance of $1 \mathrm{~m}$. While for the microstrip dipole transmitting antenna, the value of S11 is not changed with or without the presence of receiving antenna, for any distance between the transmitter and receiver antennas.

TABELA I. S11 of WiRE Dipole ANTENNA IN ANECHOIC CHAMBER

\begin{tabular}{|c|c|c|c|}
\hline \multirow{2}{*}{} & \multicolumn{3}{|c|}{ Wire Dipole Antenna (frequency 1.8 GHz) } \\
\cline { 2 - 4 } & \multicolumn{4}{|c|}{ Distance between antennas (meters) } \\
\cline { 2 - 4 } & $\mathbf{3 . 0 0}$ & $\mathbf{2 . 0 0}$ & $\mathbf{1 . 0 0}$ \\
\cline { 2 - 4 } & -36.65 & -37.11 & -31.49 \\
\hline $\begin{array}{c}\text { Receiving antenna } \\
\text { load short circuit }\end{array}$ & -35.97 & -35.52 & -31.67 \\
\hline $\begin{array}{c}\text { Receiving antenna } \\
\text { matched load }\end{array}$ & \multicolumn{3}{|c|}{-39.5} \\
\hline $\begin{array}{c}\text { Without receiving } \\
\text { antenna }\end{array}$ & \multicolumn{3}{|c}{} \\
\hline
\end{tabular}

TABELA II. S11 OF Microstrip Dipole ANTENNA IN ANECHOIC CHAMBER

\begin{tabular}{|l|c|c|c|}
\hline \multirow{2}{*}{} & \multicolumn{3}{|c|}{ Wire Dipole Antenna (frequency 900 MHz) } \\
\cline { 2 - 4 } & \multicolumn{4}{|c|}{ Distance between antennas (meters) } \\
\cline { 2 - 4 } & $\mathbf{3 . 0 0}$ & $\mathbf{2 . 0 0}$ & $\mathbf{1 . 0 0}$ \\
\cline { 2 - 4 } & -18.08 & -18.80 & -18.56 \\
\hline $\begin{array}{l}\text { Receiving antenna } \\
\text { load short-circuit }\end{array}$ & -18.37 & -18.75 & -18.54 \\
\hline $\begin{array}{l}\text { Receiving antenna } \\
\text { matched load }\end{array}$ & \multicolumn{3}{|c|}{-18.91} \\
\hline $\begin{array}{l}\text { Without receiving } \\
\text { antenna }\end{array}$
\end{tabular}

It can be observed from the results presented in Tables 1 and 2 that the S11 values of the two transmitting antennas used in the experiment, hardly varied in the cases of receiving antennas with matched load and load short-circuit, contrary to what is expected in theory transmission lines, which are expected to have an antenna impedance matching with the load should present no reflection waves, that is, the VSWR should be equal to 1 . In the case of wire dipole transmitting antenna, also noted that the experiment with the receiving antenna matched load, the value of S11 is larger than the value of the S11 in the absence thereof. Still, compared to previously published works with measurements of RFID tag [11], [13], [14], [15], wire dipole antenna and microstrip dipole are not good signs for transponder.

\section{CONCLUSION}

We presented an experimental method of measuring backscattering signal using a single port network analyzer connected to an antenna transmitting in an anechoic chamber with an without receiving antenna inside. Measurements were performed using two types of dipole antennas. The experimental results showed that the receiving antenna dipole wire showed a better reflection of the backscattered signal compared to the microstrip dipole. The dipoles studied are not a good indication for a backscatter radio links. In future work, new antenna design will be studied for use in chipless RFID systems seeking advantages over the antennas currently used.

\section{ACKNOWLEDGMENT}

The authors express their gratitude to CAPES for financial and technical support.

\section{REFERENCES}

[1] D. M. Dobkin, The RF in RFID: Passive UHF RFID in Practice, Newnes, 2007.

[2] J. D. Griffin and G. D. Durgin, "Complete Link Budgets for Backscatter Radio and RFID Systems", IEEE Antennas and Propagation Magazine, vol. 51, $\mathrm{n}^{\circ}$ 2, pp. 11-25, April 2009.

[3] G. Vannucci; A. Bletsas and D. Leigh, "A Software-Defined Radio System for Backscatter Sensor Networks", IEEE Transactions on Wireless Communications, vol. 7, nº 6, pp. 2170 - 2179, June 2008.

[4] E. Perret; M. Hamdi; A. Vena; F. Garet; M. Bernier; L. Duvillaret and S. Tedjini, "RF and $\mathrm{THz}$ Identification Using a New Generation of Chipless RFID Tags", Radioengineering Journal, vol. 20, n 2, pp. $380-$ 386, June 2011.

[5] J. Wang; H. Hassanieh; D. Katabi and P. Indyk, "Efficient and Reliable Low-Power Backscatter Networks". In: ACM SIGCOMM, Helsinki Finland, August 2012.

[6] Z. N. Chen; “Antennas for RFID Tags and Readers". In: IEEE AP/MTT HK Joint Chapter, May 2010.

[7] I. Balbin and N. C. Karmarkar; "Phase-Encoded Chipless RFID Transponder for Large-Scale Low-Cost Applications", IEEE Microwave and Wireless Components Letters, vol. 19, $\mathrm{n}^{\circ} 8$, pp. 509-511, August 2009.

[8] I. Jalaly and I. D. Robertson, "Capacitively tuned microstrip resonators for RFID barcodes,”. In Proc. 35th EUMC’05, Paris, France, , pp. 11611164, October 2005.

[9] S. Shrestha; M. Balachandran; M. Agarwal; V. V. Phoha and K. Varahramyan, "A Chipless RFID Sensor System for Cyber Centric Monitoring Applications", IEEE Transactions on Microwave Theory and Techniques, vol. 57, nº 5, May 2009.

[10] S. Preradovic; I. Balbin; N. C. Karmakar and G. F. Swiegers, "Multiresonator Based Chipless RFID System for Low-Cost Item Tracking", IEEE Transactions on Microwave Theory and Techniques, vol. 57, $\mathrm{n}^{\circ}$ 5, May 2009.

[11] C. A. Balanis, Teoria das antenas: análise e síntese, vol. 1, $3^{\mathrm{a}}$ edição, LTC, Rio de Janeiro, 2005.

[12] R. Bansal, "The Far-field: How Far is Far Enough?”, Applied Microwave and Wireless, November 1999.

[13] P. V. Nikitin and K. V. S. Rao, "Theory and Measurement of Backscattering from RFID Tags", IEEE Antennas and Propagation Magazine, vol. 48, n 6, pp. 212-218, December 2006.

[14] T. Li and D. Wang, "Experimental Studying Measurement Metrics of RFID System Performance". In $3^{\text {rd }}$ International Conference on Anticounterfeiting, Security and Identification in Communication, pp. 233237, August 2009

[15] P. V. Nikitin and K. V. S. Rao, "Measurement of Backscattering from RFID Tags", In Proceedings of Antennas Measurement Techniques Association Symposium, Newport, October 2005. 